\title{
Ecohydraulic-driven Real-Time Control of Stormwater Basins
}

\author{
D. Muschalla ${ }^{*}$, G. Pelletier* ${ }^{*}$,E. Berrouard**, J.-F. Carpenter ${ }^{* *}$, \\ B. Vallet ${ }^{* *}$ and P.A. Vanrolleghem* \\ * modelEAU, Université Laval, 1065 Avenue de la Médecine, Québec, QC, G1V 0A6, Canada (E- \\ mail:dirk.muschalla@gci.ulaval.ca; peter.vanrolleghem@gci.ulaval.ca) \\ ** Département de génie civil, Université Laval, 1065 Avenue de la Médecine, Québec, QC, \\ G1V0A6, Canada (E-mail:genevieve.pelletier@gci.ulaval.ca,emilie.berrouard.1@ulaval.ca, \\ jason-faber.carpenter.1@ulaval.ca,bertrand.vallet.1@ulaval.ca,)
}

\begin{abstract}
Control of stormwater basins can be a competitive measure to improve the ecohydraulics of urban rivers by increasing the removal efficiency of particulates and agglomerated contaminants like heavy metals and decrease hydraulic peak flows. In this paper, we present a simulation study that evaluates the potential of ecohydraulic driven real-time control of stormwater basins to improve water quality and river morphology. Thirteen different static and dynamic control scenarios were analyzed based on a detailed hydraulic and quality model of a small urban catchment equipped with a stormwater basin at its outlet. The removal efficiency for suspended solids could be significantly increased by all control strategies. At the same time, the hydraulic peaks were reduced by at least $50 \%$. The developed dynamic control strategies proved to be advantageous as they provide significant higher removal efficiency for suspended solids and a possible flexible adaptation to future demands.
\end{abstract}

\section{KEYWORDS}

Hydraulic stress; heavy metals; RTC; stormwater pond; sedimentation; mosquito control

\section{INTRODUCTION}

It is well known that urban stormwater has serious impacts on receiving waters including water quality degradation, flow-induced erosion, and habitat loss (Novotny and Witte 1997; Walsh 2000; Pitt 2002).

The objective of this contribution is to apply control strategies in stormwater management to reduce the impact on local aquatic ecosystems. This is achieved by equipping stormwater basins with dynamic sluice gates (Jacopin et al. 1999; Middleton and Barrett 2008). Our major aims are to increase the water retention time in a basin and to decrease hydraulic peaks to the receiving river. The first objective increases sedimentation and thus enables retention of major contaminants such as heavy metals and phosphorous which are agglomerated onto fine particles (Characklis and Wiesner 1997; Pettersson 2002; Vaze and Chiew 2004). The second objective focuses on reducing the hydraulic stress in the receiving river by avoiding peak discharges (Booth and Jackson 1997; Borchardt and Sperling 1997). The challenge resides in adjusting the basins' sluice gate to retain stormwater as long as required for sedimentation of fine particles without causing overflow of the basins and damages to neighboring structures when the next rainfall occurs.

The focus of this paper is to evaluate control strategies for the dynamic sluice gates to improve retention of major contaminants and prevent peak discharges, thus improving ecohydraulics in the receiving water. Starting from a case study for a small urban catchment with one stormwater basin, the proposed control strategies are evaluated in view of increasing the removal efficiency of fine particles and decreasing hydraulic peaks. 


\section{METHODS}

The analysis is conducted in three steps. Within the first step, a model for the hydraulics and quality aspects of the urban catchment is developed. Using this model, the second step aims at developing a static control of the stormwater basin by adjusting the maximum outflow of the basin. The final step suggests a set of control rules that regulate the outflow in real time.

\section{Case study}

A stormwater basin from Quebec City, Canada is selected for the case study (Figure 1). It is located in a new residential area, equipped with a dual drainage sewer system for stormwater. Dual drainage systems utilize the stormwater sewer network and city streets as dual conveyance pathways. For a thorough discussion of this topic, refer to Smith (2006). The catchment comprises 264 single-family homes and some apartment complexes on the southern border. The estimated population is 917 . The total area is $15.3 \mathrm{ha}, 30 \%$ of which is impervious. The minor stormwater sewer was designed based on a synthetic rain event with 1-hour duration and a 2-year return period and a 1-hour, 100-year synthetic rain event for the major sewer system and the stormwater basin. The stormwater basin was designed with a retention volume of $3300 \mathrm{~m}^{3}$ and a maximal water level of $1.4 \mathrm{~m}$ at the outlet. Table 1 summarizes the calculated stormwater flow at the system's outlets. Figure 1 shows an aerial photograph and the drainage network of the study site (left) and photographs of the inflow and outflow structures of the retention basin during a 3 to 5 -year event.

Table 1. Flow calculated for the sewer systems

\begin{tabular}{lcccc}
\hline Outlet from & \multicolumn{2}{c}{ Minor sewer system } & Major sewer system & Basin (controlled) \\
\hline Return period rain event & 2 year & 100 year & 100 year & 100 year \\
\hline Flow $\left(\mathrm{m}^{3} / \mathrm{s}\right)$ & 0.72 & 0.89 & 1.89 & 0.40 \\
\hline
\end{tabular}

\section{Models}

\section{Hydraulic model}

The hydrological and hydraulic model for the catchment including the stormwater basin is based on the standard models available in SWMM5 (EPA 2008) using a non-linear reservoir concept, Manning's equation and Horton infiltration model for surface runoff and the full St. Venant equations for flow routing in open channels and closed conduit systems. Data on geography, land use, and geometry of the sewer systems was available in high spatial resolution and quality. This enabled us to develop a detailed model to characterize the expected hydraulic behavior of the catchment adequately.

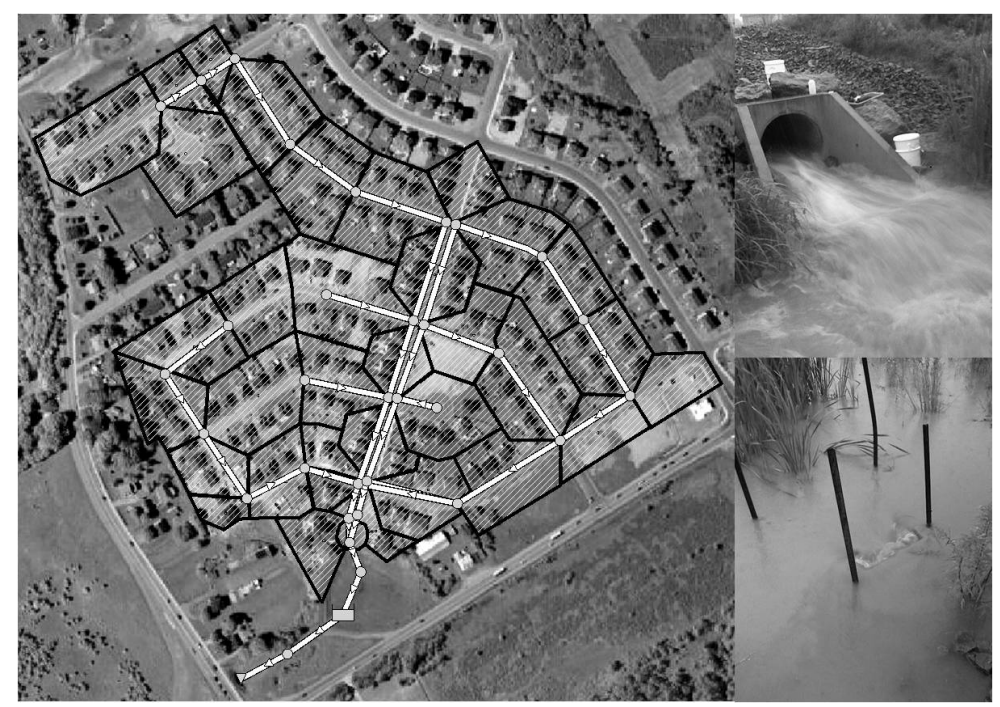

Figure 1. Overview of the study area with structure of the SWMM model (left); inflow (above) and outflow (below) of the stormwater basin for the August $18^{\text {th }} 2008$ rain event. 


\section{Quality model}

The quality model consists of three sub models; a build-up and a wash-off model for the surface runoff and a sedimentation model for the stormwater basin. We limit the water quality study to total suspended solids (TSS). As the contaminants of interest are attached to the fine particles, different particle size fractions were defined for the stormwater basin quality model.

For the build-up model, an exponential asymptotic build-up equation described by Alley and Smith (1981) was used (equation 1).

$$
\frac{d M a}{d t}=A C C U-D I S P \cdot M a
$$

Where $M a$ is the accumulated mass $(\mathrm{M})$ at time $\mathrm{t}, A C C U$ is the accumulation rate $\left(\mathrm{M} \mathrm{T}^{-1}\right)$ and DISP is the dispersion coefficient $\left(\mathrm{T}^{-1}\right)$. The wash-off was calculated according to the model developed by Metcalf and Eddy (1971) that is further described by Alley (1981) (equation 2).

$$
\frac{d M a}{d t}=-K e \cdot r(t) \cdot M a
$$

Where $r(t)$ is the runoff rate $\left(\mathrm{L} \mathrm{T}^{-1}\right)$ at time $t$ and $K e$ is the wash-off coefficient $\left(\mathrm{L}^{-1}\right)$. As quality model for the stormwater basin, the Camp model for dynamic settling (Fair and Geyer 1954; EPA 1986) was chosen (equation 3). This approach has the advantage that it is adjustable to different degrees of turbulence within the basin. A comprehensive discussion of available models for solid removal can be found in Kutzner et al. (2007).

$$
\eta_{d}=1-\left(1+\frac{1}{n} \cdot \frac{V_{s}}{v_{0}}\right)^{-n}
$$

Where $\eta_{d}$ is the dynamic settling removal efficiency, $n$ the pond settling performance factor (turbulence factor), $V_{s}$ the settling velocity $\left(\mathrm{L} \mathrm{T}^{-1}\right), v_{0}$ the surface loading rate $\left(\mathrm{L} \mathrm{T}^{-1}\right) . n$ is in the range from 1 (very poor conditions) to 3 (good conditions) up to very large values for ideal conditions for settling (low turbulence). The surface loading rate was calculated as follows:

$$
v_{0}=\frac{Q}{A_{p}}
$$

where $Q$ is the flow $\left(\mathrm{L}^{3} \mathrm{~T}^{-1}\right)$ and $A_{p}$ the pond surface $\left(\mathrm{L}^{2}\right)$. If $n$ becomes very large (ideal conditions) equation (3) can be reduced to equation 5 :

$$
\eta_{d, \text { ideal }}=1-e^{-\frac{V_{s}}{v_{0}}}
$$

This study uses settling velocities for different particle sizes as provided by MOEE (1994). These velocities have been estimated from stormwater particle size distributions, which were compiled in the NURP (Nationwide Urban Runoff Program, (EPA 1983)) study. It should be noted that these settling velocities (see table 2) are much lower than settling velocities calculated by Stokes' Law. Stokes' Law assumes ideal settling conditions and spherical particles. Neither of these assumptions normally holds in experimental studies. Additionally, the particle size distribution (PSD) is highly site specific and varies from rain event to rain event and even within a single event (Jacopin et al. 1999). Two different PSDs are used in this study. The first one was based on findings from the NURP and described in MOEE (1994), the second one was obtained from a measurement campaign accompanying this study (see table 2). The model for the original state was developed, the hydraulic behavior was analyzed and the quality model for the TSS fractions was calibrated with data obtained from the single precipitation event (August $18^{\text {th }} 2008$ ). 
Table 2. Average settling velocity for different particle size ranges based on MOEE (1994), fraction of total mass based on MOEE (1994) and measured at case study site.

\begin{tabular}{ccccc}
\hline $\begin{array}{c}\text { Size } \\
\text { fraction } \\
\mathrm{i}\end{array}$ & $\begin{array}{c}\text { Particle size } \\
\text { range }(\mu \mathrm{m})\end{array}$ & $\begin{array}{c}\text { Average settling } \\
\text { velocity of particles in } \\
\text { size fraction } \mathrm{i} \\
\mathrm{V}_{\mathrm{s}, \mathrm{i}}(\mathrm{m} / \mathrm{s})\end{array}$ & $\begin{array}{c}\text { Fraction of total mass } \\
\text { contained in size } \\
\text { fraction i }(\%) \text { - MOEE }\end{array}$ & $\begin{array}{c}\text { Fraction of total mass } \\
\text { contained in size } \\
\text { fraction i }(\%)- \\
\text { measured }\end{array}$ \\
\hline 1 & $\mathrm{x} \leq 20$ & $2.54 \mathrm{E}-06$ & 20 & 83.4 \\
2 & $20 \leq \mathrm{x} \leq 40$ & $1.30 \mathrm{E}-05$ & 10 & 9.1 \\
3 & $40 \leq \mathrm{x} \leq 60$ & $2.54 \mathrm{E}-05$ & 10 & 4.4 \\
4 & $60 \leq \mathrm{x} \leq 130$ & $1.27 \mathrm{E}-04$ & 20 & 4.1 \\
5 & $130 \leq \mathrm{x} \leq 400$ & $5.93 \mathrm{E}-04$ & 20 & - \\
6 & $400 \leq \mathrm{x} \leq 4000$ & $5.50 \mathrm{E}-03$ & 20 & - \\
\hline
\end{tabular}

\section{Control strategies and rules}

The developed control strategies are based on the following objectives and constraints:

- decrease TSS loads and agglomerated contaminant loads to the receiving water by increasing the time for sedimentation;

- decrease hydraulic peak impacts by limiting the maximal outflow of the stormwater basin;

- avoid overflows of the stormwater basin to prevent flooding or damage to structures;

- consider the length of aquatic life stages of mosquitoes as limiting factor for the time span water can be stored in the basin (for possible mosquito control strategies in constructed wetlands see e.g. Knight et al. (2003)).

For the control strategy, two different approaches were pursued, a static and a dynamic control. The static control aims to adjust one optimal maximal outflow of the stormwater basin. This straightforward optimization problem can be easily solved by modifying the maximum outflow of the stormwater pond and checking the effects against the objectives and constraints by conducting a long-term simulation. For the dynamic control strategy, different rules must be derived. In our case, the rules are:

1) If a runoff event occurs, close the outlet of the stormwater basin completely, to store the water as long as possible to guarantee maximum removal efficiency;

2) If a certain water level in the stormwater basin is reached and it is still raining or starts to rain again, open the outlet to a predefined maximum set point to prevent hydraulic stress in the recipient water;

3) If a predefined maximum water level in the pond is reached, open the outlet completely to avoid overflow.

This basic set of rules was updated by introducing a mosquito prevention control rule:

4a) If the stormwater is stored longer than $80 \%$ of the aquatic life span of mosquitoes, starting from the time where the filling of the basin starts, open the outlet only up to a predefined maximum set point to prevent hydraulic stress in the recipient.

Also, a "free up storage volume" rule was introduced that aims at an optimal utilization of the available storage volume by releasing stormwater if no further settling (e.g. over 99\%) is expected.

4b) If the stormwater is stored longer than the maximum time span needed to retain $99 \%$ of the the finest particle fraction, starting from the time where the filling of the basin ends, open the outlet only up to a predefined maximum set point to prevent hydraulic stress in the recipient water.

Note that rule $4 \mathrm{a}$ and $4 \mathrm{~b}$ are incompatible since rule $4 \mathrm{~b}$ demands longer periods to store stormwater then the aquatic life cycle of mosquitoes can permit. 


\section{Scenarios}

Long-term simulations (July $1^{\text {st }}$ to August $20^{\text {th }} 2008$ - 50 days) are performed to analyze the original state and the potential of static and dynamic control to achieve the aims discussed above.

Original state. For the original state, the maximum outflow of the stormwater basin is set to $400 \mathrm{l} / \mathrm{s}$ ( $\sim 90 \mathrm{l} /\left(\mathrm{s}^{*} \mathrm{ha}\right)$ ); the TSS removal is simulated with the assumption of good conditions using $n=3$ in equation 3.

Static control (10 Scenarios). Ten scenarios are simulated to analyze the static control, each with a different maximum outflow rate between 15 l/s and 150 l/s $\left(\sim 31 /\left(\mathrm{s}^{*}\right.\right.$ ha) and $\sim 33$ l/(s*ha)); The TSS removal is simulated with the same assumptions as for the original state.

Dynamic control (3 Scenarios). Each dynamic control scenario is simulated twice with the assumption of good performance as in the previous scenarios and ideal performance for settling using equation 5.

1) Dynamic scenario 1: rules 1-3 (dyn. control ideal/good cond. basic)

The maximum water level for rule 2 is set to 1.2 meters, the maximum outflow to $0.2 \mathrm{~m}^{3} / \mathrm{s}$; for rule 3 , the maximum water level is set to 1.4 meters.

2) Dynamic scenario2: rules 1-3,4a (dyn. control ideal/good cond. mosq.)

For rule $4 \mathrm{a}$, the allowed time span to store water after beginning of filling is set to 4 days (aquatic life cycle of mosquitoes is between 4 days and a month depending on the species and temperature)

3) Dynamic Scenario3: rules 1-3,4b (dyn. control ideal/good cond. sett.)

For rule $4 \mathrm{~b}$, the time span needed for best possible TSS removal was set to 4.5 days after the end of inflow to the basin (average settling time for the smallest TSS fraction is $\sim 4$ days for a water depth of 1 meter using the values from table 2).

\section{RESULTS AND DISCUSSION}

The hydraulic simulation results for the original state are within a realistic range compared to the observed hydraulic behavior of the stormwater basin. The simulated water levels correspond well with the observed water levels during the conducted measurement campaign (results not shown). In addition, the simulated retention effect and the inflow and outflow hydrographs of the stormwater basin correspond with the expected physical behavior. The TSS inflow concentration is well captured for the precipitation event. In addition, the order of magnitude for the simulated outflow concentration fits well (see figure 2). However, the particle size distribution has a considerable influence on the simulated TSS concentrations and the removal efficiency.

\section{Original State}

The simulated removal efficiency for the original state varies between $86.0 \%$ using the PSD according to MOEE (1994) and 68.5\% using the measured PSD at the stormwater basin and $87.5 \%$ and $65.4 \%$ respectively for the long term simulation. All values are within the range of reported TSS removal efficiencies in the literature for this type of stormwater basin (Middleton and Barrett 2008). The different total removal efficiency for the PSD according to MOEE and the measured PSD is explainable by the different total mass contained in the size fractions. Only the results obtained with the PSD according to MOEE are discussed further. A summary of the simulated inand outflow TSS loads (including fractionation) as well as the removal efficiency for the original state of the stormwater basin is given in table 3 . 


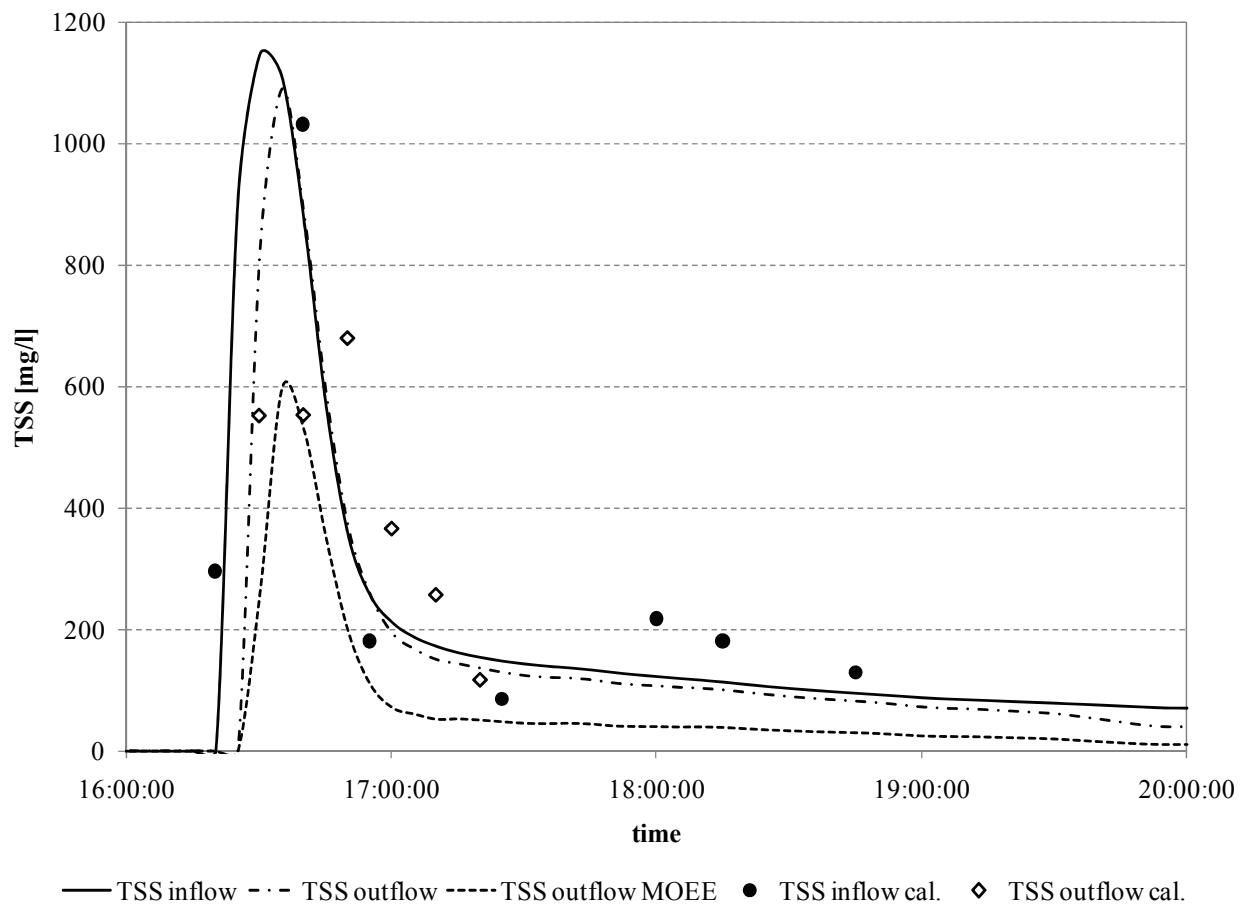

Figure 2. Simulated and measured TSS concentrations for inflow and outflow of the stormwater basin for the August $18^{\text {th }} 2008$ rain event. TSS inflow - simulated TSS inflow concentration, TSS outflow - simulated outflow concentration using PSD obtained from measurement campaign, TSS outflow MOEE - simulated outflow concentration using PSD described in MOEE (1994), TSS inflow cal. and TSS outflow cal. - TSS concentrations for inflow and outflow obtained from measurement campaign.

Table 3. Simulated removal efficiency for TSS and TSS fractions for both PSDs using the model for the original state of the stormwater basin. Short term analysis for one rain event (August $18^{\text {th }}$ 2008), long term analysis for period from $1^{\text {st }}$ July to $20^{\text {th }}$ August 2008.

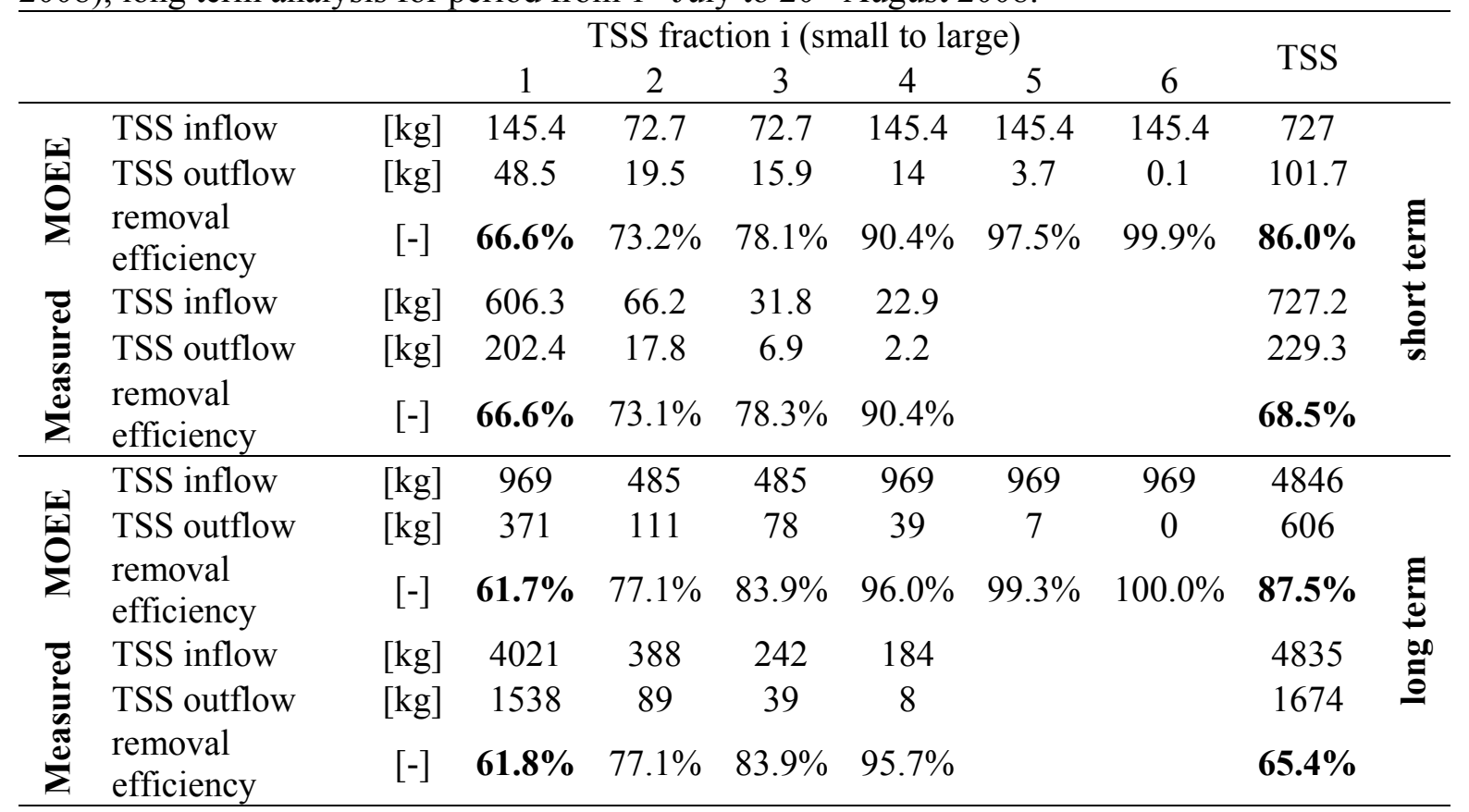




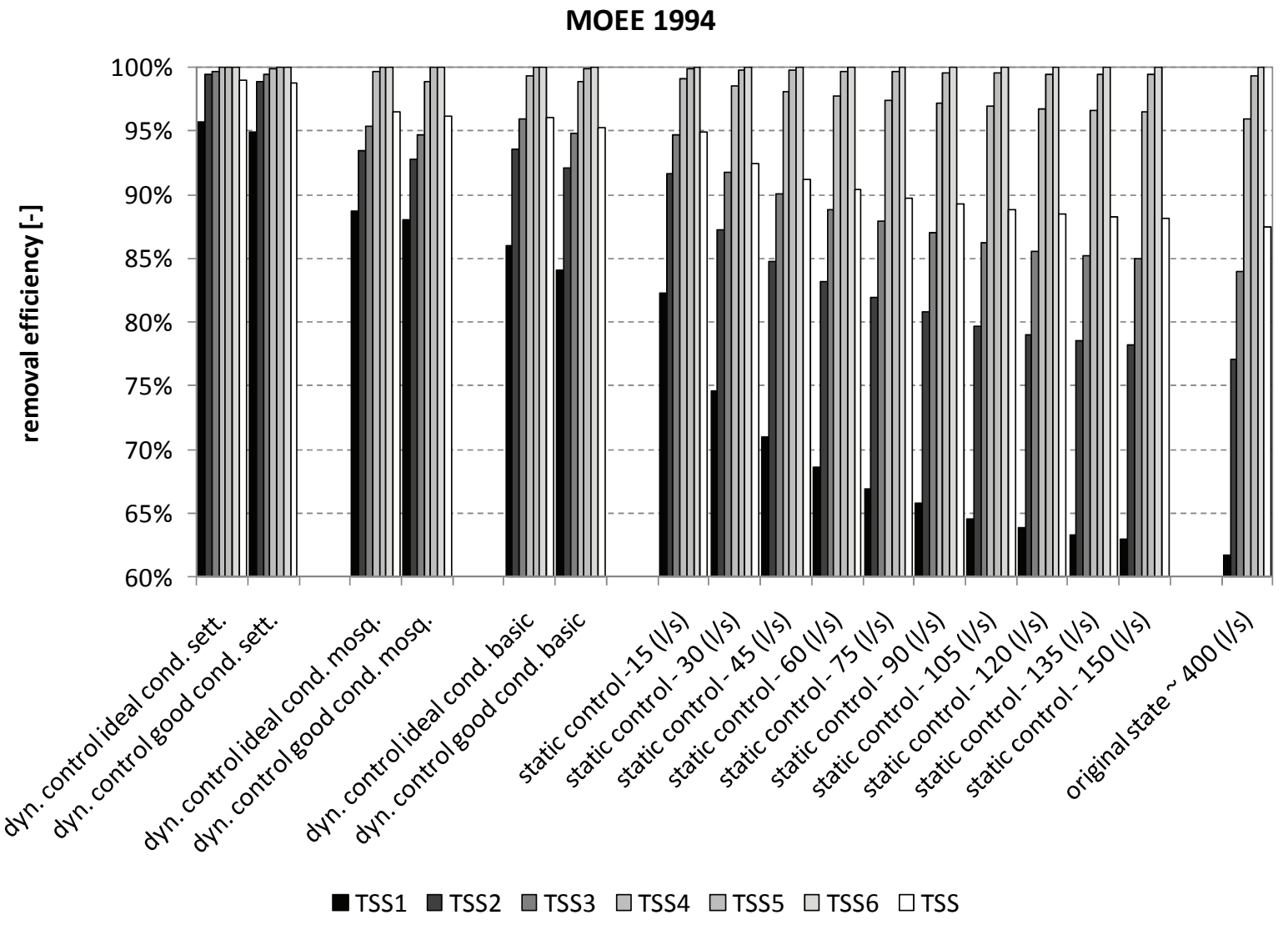

Figure 3. TSS removal efficiency for all control strategies and the original state using PSD according to MOEE (1994). TSS1 is the TSS fraction with the smallest particle size.

\section{Static Control}

TSS retention for static control is computed for maximum outflow discharges between 15 and $150 \mathrm{l} / \mathrm{s}$ (figure 3). The static control improves the overall TSS removal efficiency between $1 \%$ and $7 \%$ and the removal efficiency for the smallest particle size fraction (TSS1) from $1 \%$ up to $20 \%$ for maximum outflow of 150 and $15 \mathrm{l} / \mathrm{s}$ respectively (see figure 3 ). It is obvious that reducing the maximum outflow will increase retention time in the basin and therefore increase TSS retention. The hydraulic characteristics of the basin are not considerably changed when comparing the static control to the original state (see evolution of water depth for original state and static control $60 \mathrm{l} / \mathrm{s}$ in middle panel of figure 4). However, the design goal of the basin (retention of a 1-hour, 100-year rain event) is no longer reached under the static control scenarios resulting in unrequested more frequent emergency overflows. Note also that the reduction of the maximum outflow to very small values (e.g. $15 \mathrm{l} / \mathrm{s}$ ) is not feasible as such small outflows provoke operational problems such as clogging and time consuming emptying of the basin ( $\sim 61$ hours for the $15 \mathrm{l} / \mathrm{s}$ scenario, $\sim 2$ hours for the original design).

\section{Dynamic control}

The three dynamic control strategies perform better than all static control strategies. They improve the overall TSS removal efficiency between $8 \%$ and $12 \%$ and the removal efficiency for the smallest particle size fraction from $22 \%$ to $34 \%$ (compare figure 3). It is noted that the two extended strategies (4a) and (4b), which limit the maximum retention time in the basin, perform better than the basic dynamic control rule that allows an unlimited retention time. When analyzing the hydraulic behavior (water depth in basin; lower panel in figure 4), the reasons for this become evident. An unlimited retention time results in situations where a new rain event starts when the 
basin has not yet emptied. If the remaining storage capacity is too small, the stormwater has to be released to the recipient water body without sufficient time for an effective particle removal. Such situations are identified for the basic dynamic control strategy on day 12, 30, and 41 (see lower panel in figure 4).

The two best performing dynamic control strategies have conflicting objectives. On the one hand, the "free up storage volume" strategy results in the best removal efficiency for TSS. On the other hand, this strategy provides good conditions for reproduction of mosquitoes, reducing the quality of life of residents or even introducing a health risk. The aquatic life cycle of mosquitoes can be breached by the "mosquito control" strategy but with a considerable decrease of the TSS removal efficiency.
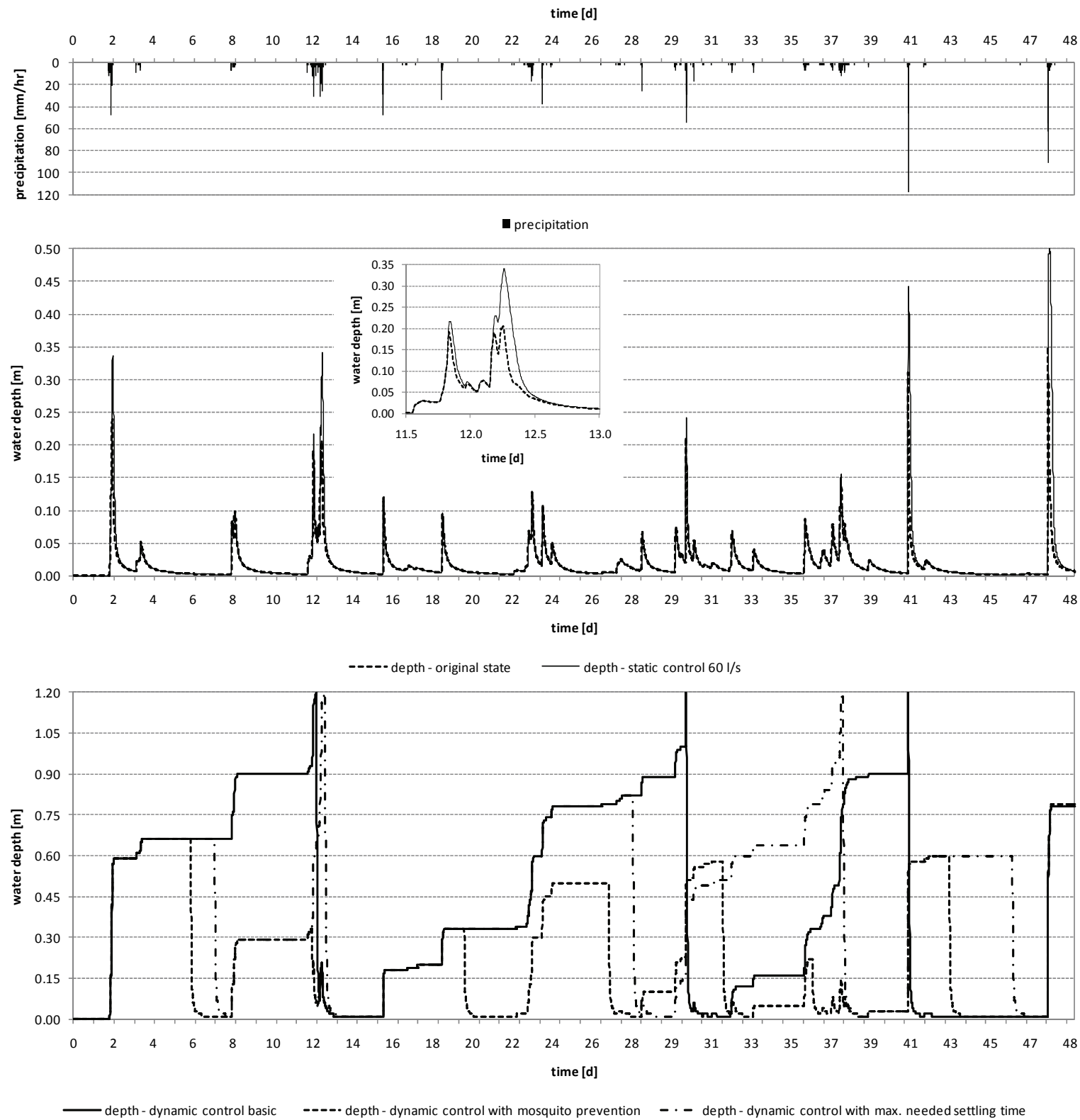

Figure 4. Hydraulic behavior (water depth) of the stormwater basin for all control strategies and the original state for the period from July $1^{\text {st }}$ to August $20^{\text {th }} 2008$. upper panel: precipitation time series; middle panel: water depth for the original state and the static control with $60 \mathrm{l} / \mathrm{s}$ maximum outflow; lower panel, the resulting water depth for the three dynamic control strategies. 
The three dynamic control strategies demand equipment that is more complex and requires more maintenance compared to the static control (i.e. higher costs). In return, the system is more flexible and the basin can be operated as dual-purpose facility: fulfilling the original design criteria for hydraulic retention and flood protection and allowing an optimized operation for pollutant control. In addition, the system becomes adaptable to future demands, e.g. climate change.

\section{CONCLUSIONS}

The simulation study showed that real-time control (RTC) of the sluice gate proves to be an effective solution for reducing the suspended solids discharge and hydraulic stress in the urban river. In all studied scenarios, the controlled basin enabled removal efficiencies for fine particles and associated contaminants such as heavy metals that were much higher than for the current basin without control. At the same time, hydraulic peaks could be considerably reduced. The dynamic control of the outlet proved advantageous over the static control, as dynamic control results in higher TSS removal efficiency, allows a multipurpose operation of the basin and a flexible adaptation to future demands. It became also clear that the design of the dynamic control rules results in a multi-objective optimization problem. Consequently, environmental aims and social impacts (e.g. removal efficiency against mosquito control) are conflicting.

\section{ACKNOWLEDGEMENTS}

The described study is part of the strategic project entitled "Integrating river ecohydraulics in urban stormwater management" funded by the Natural Sciences and Engineering Research Council of Canada (NSERC). Peter A. Vanrolleghem holds the Canada Research Chair in Water Quality Modeling.

\section{REFERENCES}

Alley, W. M. and Smith, P. E. (1981). Estimation of accumulation parameters for urban runoff quality modeling. Water Resources Research, 17(6), 1657-1664.

Alley, W. M. (1981). Estimation of impervious-area washoff parameters. Water Resources Research, 17(4), 1161-1166.

Booth, D. B. and Jackson, C. R. (1997). Urbanization of aquatic systems: Degradation thresholds, stormwater detection, and the limits of mitigation. Journal of the American Water Resources Association, 33(5), 1077-1090.

Borchardt, D. and Sperling, F. (1997). Urban stormwater discharges: Ecological effects on receiving waters and consequences for technical measures. Water Science and Technology, 36(8-9): 173-178.

Characklis, G. W. and Wiesner, M. R. (1997). Particles, metals, and water quality in runoff from large urban watershed. Journal of Environmental Engineering-ASCE, 123(8), 753-759.

EPA (1983). Results of the nationwide urban runoff program, volume 1 - final results. United States Environmental Protection Agency, Washington, DC.

EPA (1986). Methodology for analysis of detention basins for control of urban runoff quality. United States Environmental Protection Agency, Washington, DC. 
EPA (2008). Storm Water Management Model (SWMM), Version 5.0. United States Environmental Protection Agency, Cincinnati, Ohio.

Fair, G. M. and Geyer, J. C. (1954). Water supply and waste-water disposal. John Wiley and Sons, New York.

Jacopin, C., Bertrand-Krajewski, J. L. and Desbordes, M. (1999). Characterisation and settling of solids in an open, grassed, stormwater sewer network detention basin. Water Science and Technology, 39(2), 135-144.

Knight, R. L., Walton, W. E., O'Meara, G. F., Reisen, W. K. and Wass, R. (2003). Strategies for effective mosquito control in constructed treatment wetlands. Ecological Engineering 21(4-5), 211232.

Kutzner, R., Brombach, H. and Geiger, W. F. (2007). Sewer solids separation by sedimentation the problem of modeling, validation and transferability. Water Science and Technology, 55(4), 113123.

Metcalf and Eddy (1971). Storm Water Management Model - Volume 1 - Final Report. United States Environmental Protection Agency.

Middleton, J. R. and Barrett, M. E. (2008). Water quality performance of a batch-type stormwater detention basin. Water Environment Research, 80(2), 172-178.

MOEE (1994). Stormwater management practices planning and design manual. Ministry of Environment and Energy, Ontario, Canada.

Novotny, V. and Witte, J. W. (1997). Ascertaining aquatic ecological risks of urban stormwater discharges. Water Research, 31(10), 2573-2585.

Pettersson, T. J. R. (2002). Characteristics of suspended particles in a small stormwater pond. 9th International Conference on Urban Drainage, Portland, Oregon, USA.

Pitt, R. (2002). Receiving water impacts associated with urban runoff. In: Handbook of ecotoxicology, D. Hoffmann, B. Rattner, G. A. Burton and J. Cairns (ed.), CRC-Lewis, Boca Raton, FL, USA.

Smith, M. B. (2006). Comment on 'Analysis and modeling of flooding in urban drainage systems'. Journal of Hydrology 317(3-4), 355-363.

Vaze, J. and Chiew, F. H. S. (2004). Nutrient loads associated with different sediment sizes in urban stormwater and surface pollutants. Journal of Environmental Engineering-ASCE, 130(4), 391-396.

Walsh, C. J. (2000). Urban impacts on the ecology of receiving waters: a framework for assessment, conservation and restoration. Hydrobiologia 431(2-3), 107-114. 